\title{
Nanofluidics for Simultaneous Size and Charge Profiling of Extracellular Vesicles
}

\author{
Imman I Hosseini, ${ }^{\dagger}$ Zezhu Liu, ${ }^{\ddagger}$ Xavier Capaldi, ${ }^{\ddagger}$ Tamer Abdelfattah, ${ }^{\dagger}$ Laura \\ Montermini, " Janusz Rak, "Walter Reisner, ${ }^{\ddagger}$ and Sara Mahshid*,† \\ $\dagger$ Department of Biomedical Engineering, McGill University, 3775 rue university, Montreal, \\ Quebec H3A 2B4, Canada \\ $\ddagger$ Department of Physics, McGill University, 3600 rue university, Montreal, Quebec H3A \\ 2T8, Canada \\ \Department of Pediatrics, McGill University, 3775 rue university, Montreal, Quebec H3A \\ 2B4, Canada \\ E-mail: sara.mahshid@mcgill.ca \\ Phone: +1 (514) 398-8964
}

\section{Device Fabrication and Operation}

The device is fabricated with the process outlined in Capaldi et al. ${ }^{1}$ Briefly, a $400-500 \mathrm{~nm}$ deep nanoslit is fabricated in borofloat glass with UV lithography followed by a CHF3: CF4 etch step. Then, circular nanocavities with a radius of $500 \mathrm{~nm}$ are defined lithographically and etched $200 \mathrm{~nm}$ deep with RIE. Next, the substrate is bonded to a silicon wafer coated on both sides with $50 \mathrm{~nm}$ silicon nitride (Cornell, NanoScale Facility, silicon wafers $400 \mu \mathrm{m}$ thick). A lithography/RIE step is performed on the wafer top-side to open up a window for $\mathrm{KOH}$ etching. Final, $\mathrm{KOH}$ etching is performed to expose the free-standing nitride membrane over the nanocavity region. Our device is incubated with PVP (polyvinylpyrrolidone) 8\%. The 
fabricated device is mounted on a 3D printed chuck and plasmid or vesicle containing solution is introduced in the device inlet (Fig. S1). Pneumatic pressure applied to the device inlet is then used to drive plasmids or vesicles to the nanocavity region below the free-standing membrane.

a)

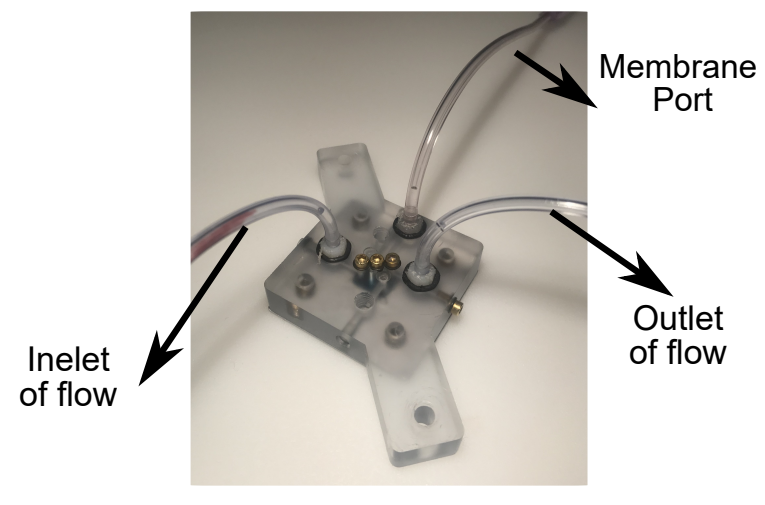

b)

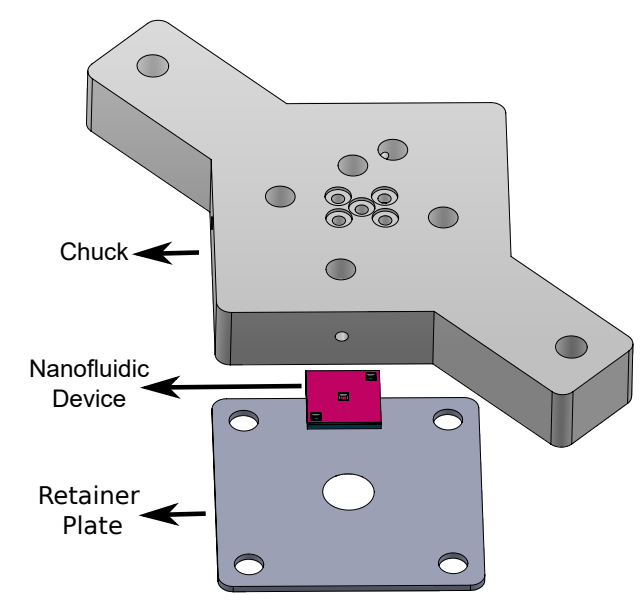

Figure 1: (a) Photograph of 3D printed chuck interfaced to luer tubing for applying pneumatic pressure to deflect membrane lid and transport solution through flow-cell. (b) Schematic showing chuck/nanofluidic device assembly.

\section{Slit Height Calibration}

In order to find the relation between the pressure used to deflect the membrane lid and the slit height $(2 h)$, we introduce fluorescent dye into the device. The variation of fluorescent dye intensity with pressure applied to the membrane lid can then be used to deduce the variation of the slit height with pressure. Consider a fluid volume $2 h A$ enclosed between the membrane lid and the device bottom surface in a region of the device of area $A$. The fluorescent intensity arising from dye molecules present in this volume is: ${ }^{2}$

$$
I_{F}=I_{e} \varepsilon(\lambda) \Phi C(2 h A)
$$


with $I_{e}$ is the light source intensity, $\varepsilon$ the molar absorptivity (a function of light source wavelength $\lambda$ ), $C$ the dye concentration and $\Phi$ the quantum efficiency of the dye. The slit height at zero applied pressure $2 h_{o}$ corresponds to a measured fluorescent intensity $I_{F o}$. From the linearity of Eq. 1, the slit height corresponding to a measured intensity $I_{F}$ is then $h=\left(I_{F} / I_{F_{o}}\right) h_{o}$. Fig. S2 gives the resulting slit height as a function of pressure applied to the membrane lid. Specifically, the reported height measurements are obtained by averaging the fluorescent dye intensity over the central portion of the membrane with a gap height varying less than $2 \delta h_{\max }$ (termed "plateau region," see inset of Fig. S8 inset). Experiments were primarily performed in devices with $\delta h_{\max }=0.8 \mathrm{~nm}$, with a corresponding "plateau region no smaller than the $6 \times 5$ array of cavities in the device center (the total membrane area was $160 \times 200 \mu \mathrm{m}^{2}$ and the slit depth $\left.h_{o}=500 \mathrm{~nm}\right)$. A second device class used a larger $\delta h_{\max }=10 \mathrm{~nm}$ (plateau region corresponded to $6 \times 6$ array of cavities in the device center, total membrane area was $100 \times 100 \mu \mathrm{m}^{2}$ and the slit depth $\left.h_{o}=400 \mathrm{~nm}\right)$.

\section{Measurement of Solution Ionic Concentration}

The electrolyte solution used is $1 \mathrm{mM}$ Tris with $0.4 \mathrm{mM} \mathrm{NaCl}$ in DI water. To find the solution ionic concentration, we measured the solution conductivity and then converted conductivity to ionic concentration. First a conductivity calibration curve is obtained using a pure $\mathrm{NaCl}$ concentration standard (i.e. conductivity of a solution of DI water and $\mathrm{NaCl}$ measured for different $\mathrm{NaCl}$ concentrations). Then, the conductivity of our experimental solution is measured and the ionic concentration obtained by comparison with the calibration curve (see Fig. S3). The conductivity of the experimental solution is $63 \frac{\mu S}{m}$ and the corresponding ionic concentration is $0.7 \mathrm{mM}$. 


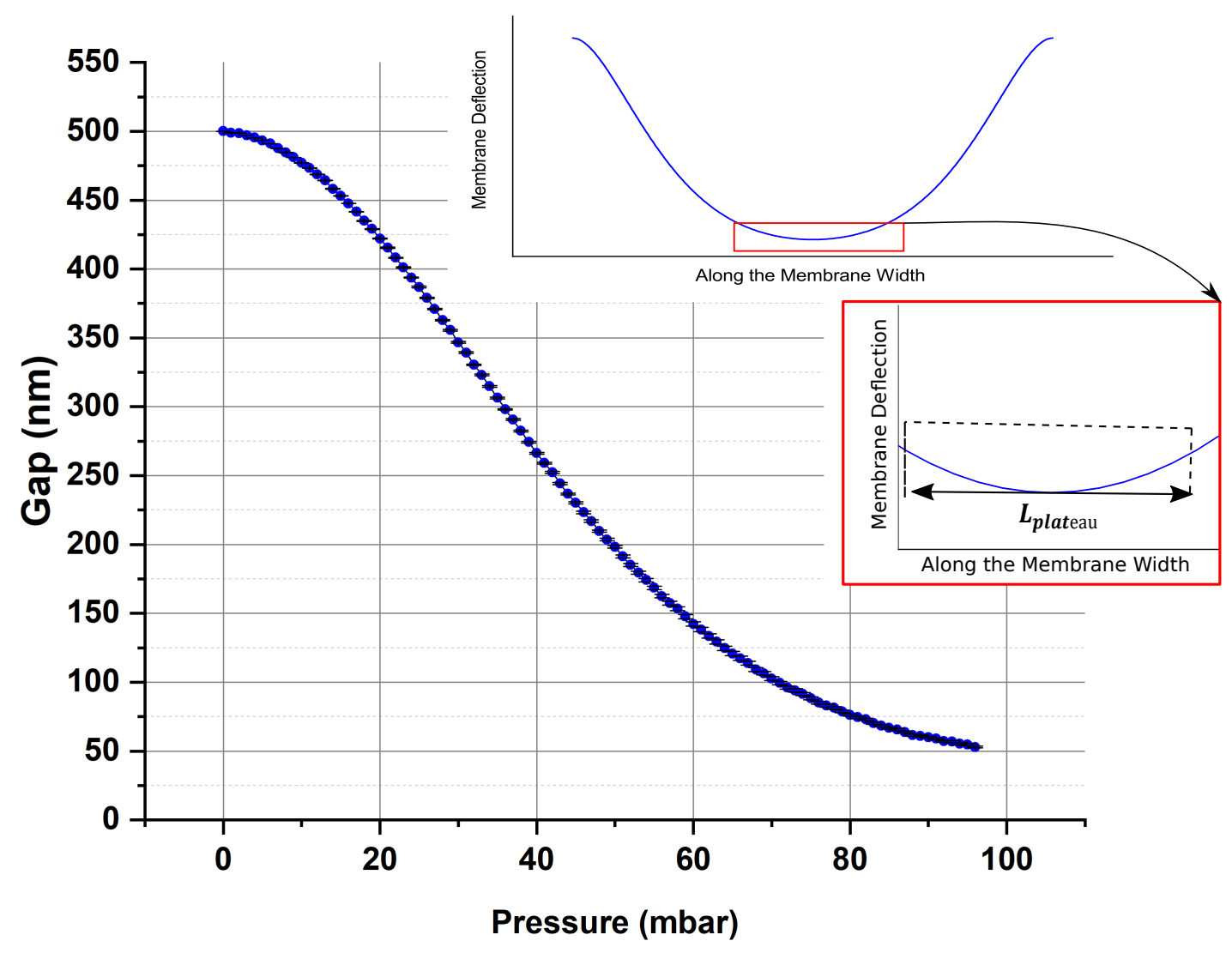

Figure 2: Relation between slit height and applied pressure used to deflect the membrane lid. The error bars show the variation of slid height in plateau region. The inset shows the plateau region definition. This curve was taken for device class 1. 


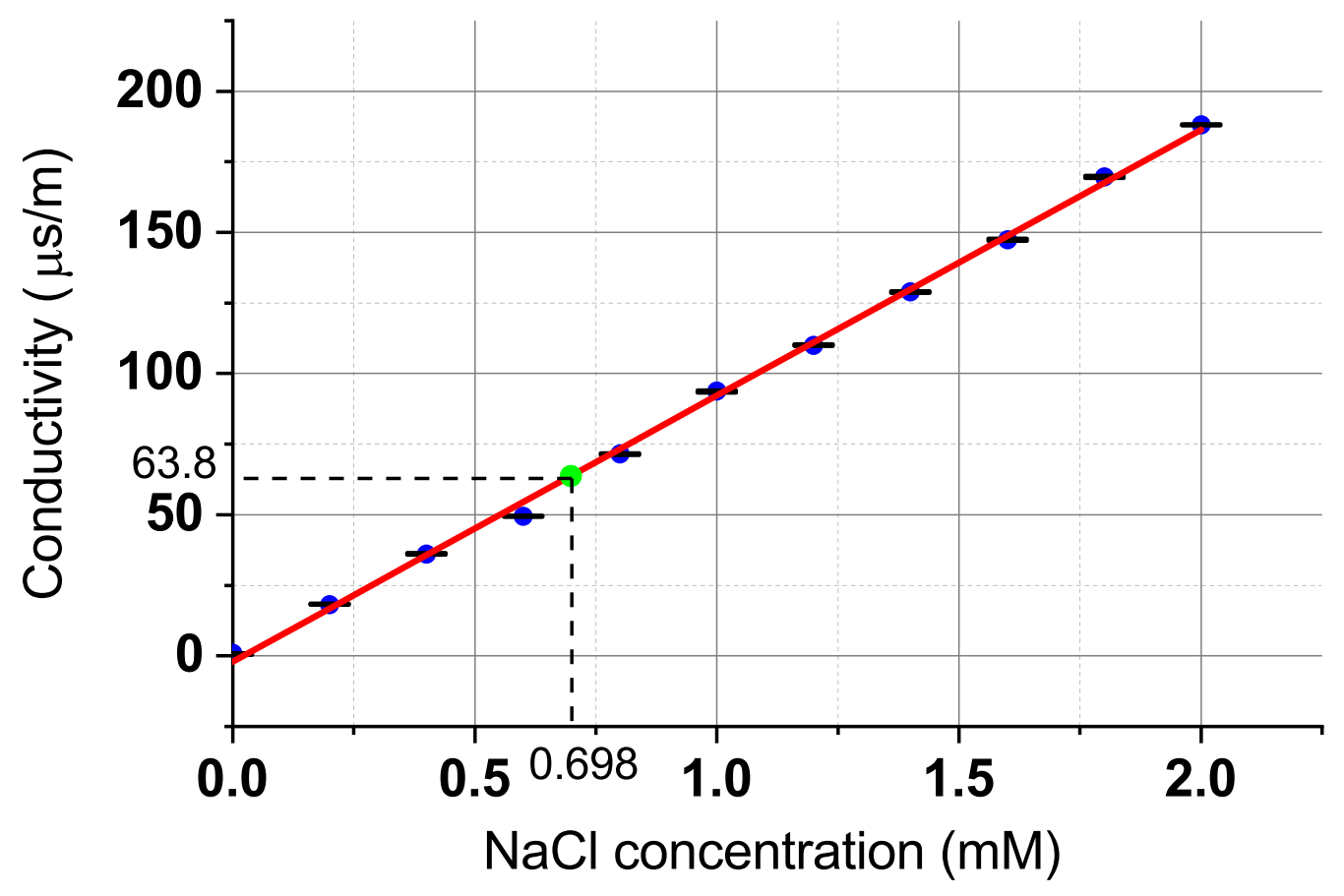

Figure 3: Solution conductivity versus ionic concentration. The error bars are smaller than the symbols and the maximum error is less than $0.1 \%$. 


\section{Calculation of Particle Escape Time}

According to Kramers' theory, for the regime $W>6 k_{B} T$, the average escape time $\left\langle t_{\text {esc }}\right\rangle$ of a particle from a potential well is given by:

$$
\left\langle t_{\mathrm{esc}}\right\rangle=t_{r} \exp \left(\frac{W}{k_{B} T}\right)
$$

in which $k_{\mathrm{B}}$ is Boltzmann constant, $T$ is temperature considered as $298 \mathrm{~K}$. The quantity $t_{r}$ is the particle positional relaxation time and $W$ is the potential well depth (see Fig. 4). Note that $t_{r}$ depends on the particle diffusion coefficient $(D)$ and the well geometry. In particular, $t_{r}=\frac{L^{2}}{4 D}$, with $L$ a length scale that depends on the cavity depth $(d)$ and cavity radius $\left(R_{\mathrm{c}}\right)$. We find that in our system $L \sim 100 \mathrm{~nm}$; the cavity depth and cavity radius are $200 \mathrm{~nm}$ and $500 \mathrm{~nm}$, respectively. To correctly relate the experimentally measured time scale $\left(\left\langle t_{\mathrm{esc}}\right\rangle\right)$ to simulation results, we introduce an "escape boundary" consisting of a radial contour at $r_{\mathrm{esc}}$ centered on the cavity $\left(r_{\text {esc }}>R_{\mathrm{c}}\right)$. The use of an escape boundary larger than the cavity radius ensures that particle escape from a well always results in the particle transitioning to a neighboring well. For analysis of both experimental results and simulation, $r_{e s c}=750 \mathrm{~nm}$ (Fig. S4a). The particle is considered to have entered the cavity when the particle radial coordinate $r \leq r_{\text {esc }}$ (occurring at time $t_{1}$ ). The particle is considered to have left the cavity when its radial coordinate $r>r_{\mathrm{esc}}$ (occurring at time $t_{2}>t_{1}$ ). The escape time $t_{\mathrm{esc}}=t_{2}-t_{1}$.

In the case that the well geometry $\left(R_{\mathrm{c}}, d\right)$ and particle chemistry remain unchanged (i.e. $q_{\text {eff }}$ is constant), $\left\langle t_{\text {esc }}\right\rangle$ depends only on the potential well depth $(W)$. We can use the dependence of $\left\langle t_{\text {esc }}\right\rangle$ on $W$ to measure the particle effective charge. The potential well depth, $W$, depends on the particle effective charge $q_{\text {eff }}$ via $W=q_{\text {eff }} \delta \psi_{\mathrm{m}}$, where $\delta \psi_{\mathrm{m}}$ is the difference of the value of the electrical potential averaged over the particle surface inside and outside of the cavity. Therefore, when the average escape time is measured for two differing degrees 
of confinement $\left(\left\langle t_{\mathrm{esc}, 1}\right\rangle\right.$ and $\left.\left\langle t_{\mathrm{esc}, 2}\right\rangle\right)$, Eq. 2 can be rewritten:

$$
q_{\mathrm{eff}}=\frac{\ln \left(\frac{\left\langle t_{\mathrm{esc}, 1}\right\rangle}{\left\langle t_{\mathrm{esc}, 2}\right\rangle}\right)}{\psi_{\mathrm{m} 1}-\psi_{\mathrm{m} 2}}
$$

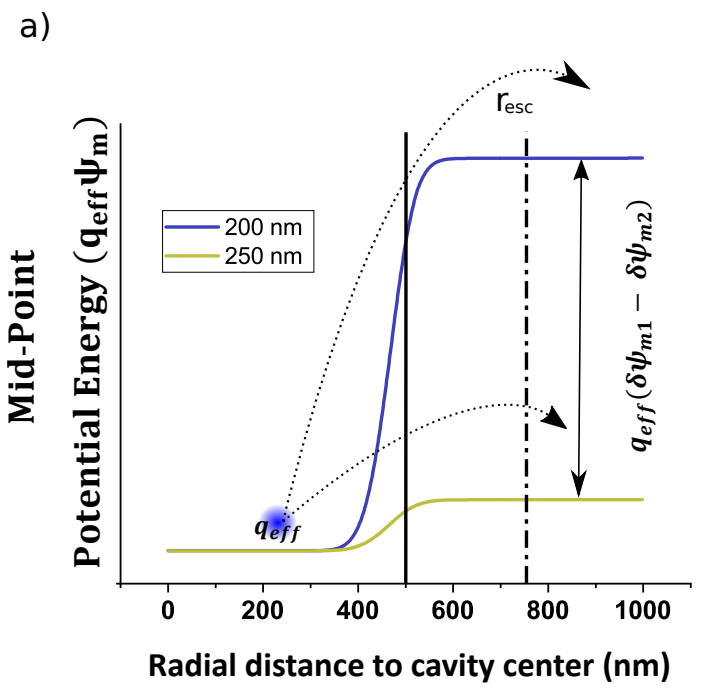

b)

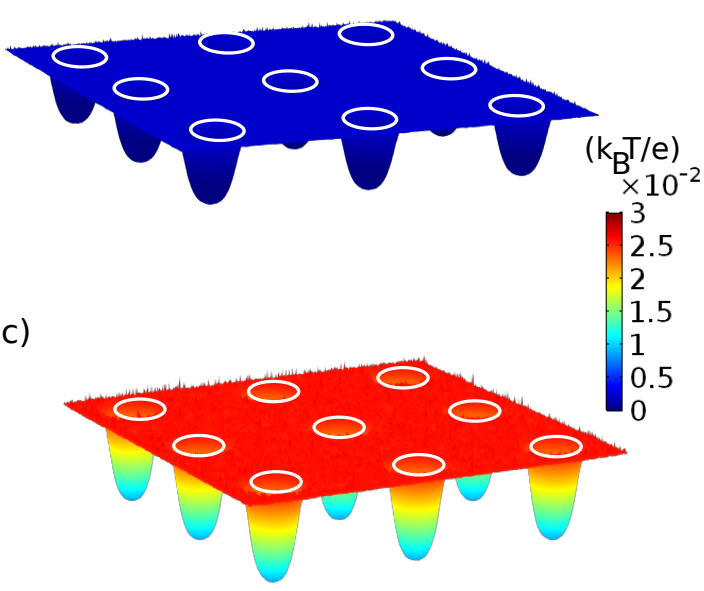

Figure 4: (a) The mid-point potential Energy as a function of radial distance from the cavity center for a slit height of $200 \mathrm{~nm}$ and $250 \mathrm{~nm}$. By decreasing the slit height, the energy barrier increases and the average of escape time increases. The quantity $r_{\text {esc }}$ gives the boundary a particle should pass to be considered as having escaped from the well. The mid-point potential corresponding to a $3 \times 3$ cavity lattice for a slit height of (b) $250 \mathrm{~nm}$ and (c) $200 \mathrm{~nm}$.

\section{Solution for Electrostatic Potential}

The electrostatic potential in the device is obtained from solution of the Poisson-Boltzmann equation:

$$
\nabla^{2} \psi=\kappa^{2} \sinh \psi
$$

here $\kappa$ is the inverse Debye length, given by $\kappa=\sqrt{\frac{2 c N_{A} e^{2}}{\varepsilon_{m} \varepsilon_{0} k_{\mathrm{B}} T}}$ with $N_{A}$ the Avagadro number, $e$ the elementary charge, $\varepsilon_{0}$ the permittivity of free space, $\varepsilon_{m}$ the relative permittivity of our medium (taken to have a value of 78 for water at a temperature $298 \mathrm{~K}$ ) and $c$ the medium ionic concentration. Eq. 4 is solved by simulation in COMSOL Multi-physics using axisymmetric 
cylindrical coordinates (r, z) according to M. Krishnan. ${ }^{3}$ (Fig. 5a,b). The particles will tend to occupy the position of minimum electrostatic potential, which occurs at mid-plane (e.g. position equidistant between membrane lid and substrate). Since the particle's size is comparable to the slit height, we need to take into account the variation of the potential around the particle. This is accomplished by computing the average of the potential over the particle's surface. ${ }^{3}$ Fig. S5c gives the dependence of the surface-averaged value of the electrostatic potential at mid-plane $\psi_{m}$ on particle radius for a slit height of $200 \mathrm{~nm}$. Note that the surface-averaged value of the electrostatic potential increases exponentially with particle radius.

a)

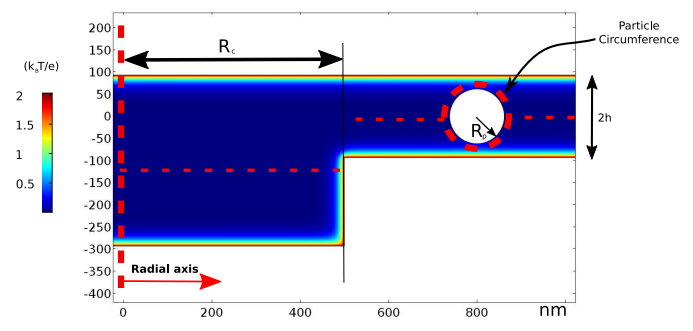

b)

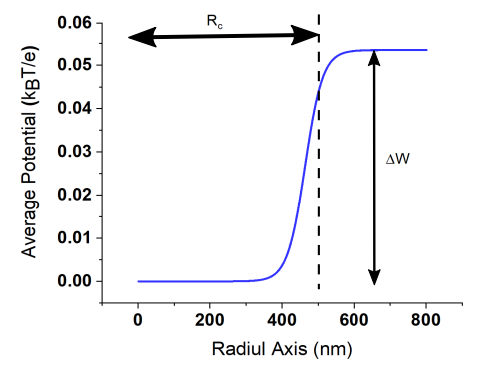

c)

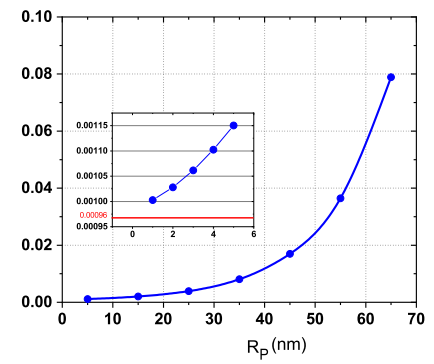

Figure 5: Electrostatic potential obtained from COMSOL simulation. (a) Electrostatic potential contours inside the chamber; (b) surface-averaged value of electrostatic potential at mid-plane $\psi_{m}$ versus the radial distance from cavity center; (c) surface-averaged value of electrostatic potential versus particle radius.

\section{Determining Zeta-Potential of Device Surfaces}

Solution of the electrostatic potential via Eq. 4 requires knowledge of the electrostatic potential (zeta-potential) on the membrane lid and substrate $\left(\psi_{\mathrm{s}}\right)$. In order to measure $\psi_{\mathrm{s}}$, we use YOYO-1 labeled pBR322 Vector as a calibration particle with known size and effective charge. The advantage of using this plasmid vector is that the plasmids, each having the same sequence, are chemically uniform. First, the average escape time of the plasmid from an electrostatic well is measured in our nanofluidic device at a specific slit height $2 h$ 
(specifically, for a slit height $2 h=185 \mathrm{~nm}$, the averaged plasmid escape time over 351 escape events is $1.85 \mathrm{~s}$, Fig. S6a). Then, using simulation, the average escape time is obtained for different $\psi_{\mathrm{s}}$ values for a particle with the same size and effective charge as the plasmid at the same fixed slit height. Note that the plasmid hydrodynamic radius is reported as $65 \mathrm{~nm}^{4}$ and the particle's effective charge is evaluated using electrophoresis as $-152 \pm 4 e$. Finally, by correlating our experimental and simulation results, the $\psi_{\text {s }}$ value yielding the same average simulated escape time as experiment is obtained. This value of $\psi_{\mathrm{s}}$ corresponds to the device zeta-potential.

a)

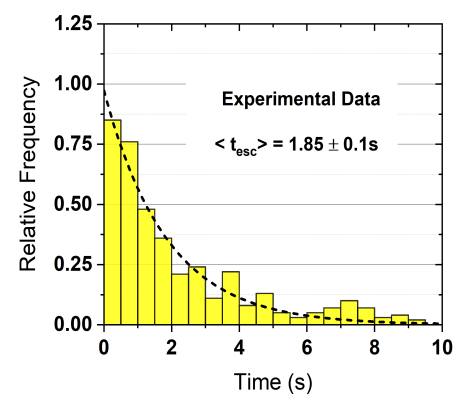

b)

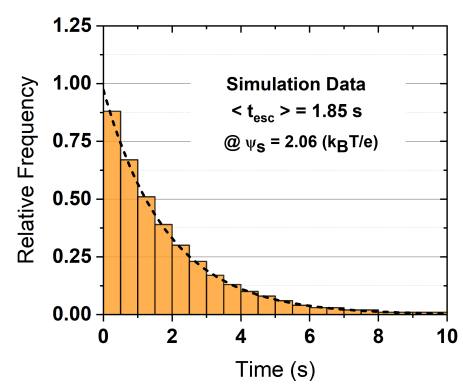

c)

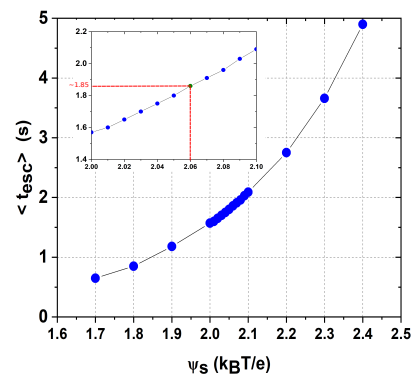

Figure 6: (a) Histogram of 351 experimentally measured escape times for pBR322 Vector with a hydrodynamic radius of $65 \mathrm{~nm}$ and a slit height of $187 \mathrm{~nm}$. (b) Histogram of 10000 simulated escape times using BD simulation for a particle with the same size, charge and the same slit height as experiment. Setting $\psi \sim 2.06\left(\frac{k_{B} T}{e}\right)$ yields an average escape time in agreement with experiment. (c) Average escape time versus surface electrostatic potential using BD simulation.

The simulated escape times are found from solving the Langevin equation:

$$
x(t+\delta t)=x(t)-\mu \nabla \bar{W}+\sqrt{2 D \delta t} w(t)
$$

Here, $D$ is the diffusion coefficient calculated in the next section. The quantity $w(t)$ is a random displacement arising from Gaussian distributed thermal fluctuations: $\langle w(t)\rangle=0$ and $\left\langle w(t) w^{T}\left(t^{\prime}\right)\right\rangle=\mathbf{I}$ if $\left|t-t^{\prime}\right|<\delta t, \mathbf{0}$ otherwise. The quantity $\mu=\frac{\delta t}{6 \pi \eta R}$. For good convergence, the simulation time step $(\delta t)$ is set at $10 \mu s$. The quantity $\bar{W}(x(t))$ is a potential energy given by $\bar{W}=q_{\mathrm{eff}} \psi_{\mathrm{m}}(x(t))$. 
The simulations are performed with the particle initially located at the cavity center. Escape occurs when the particle's radial position exceeds $r_{\text {esc }}$. Equivalent escape simulations are performed 10000 times, giving rise to a distribution of simulated escape times. To obtain the average simulated escape time, the histogrammed escape times are fitted to the same exponential model as experiment: $P(\Delta t)=A /\left\langle t_{\text {esc }}\right\rangle \exp \left(-\Delta t /\left\langle t_{\text {esc }}\right\rangle\right)$ (Fig. 6b). We performed this simulation for different surface electrostatic potential values, and the average escape time as a function of $\psi_{\mathrm{s}}$ is shown in Fig. S6c.

We found that when $\psi \sim 2.06\left(\frac{k_{B} T}{e}\right)$, there is less than a $1 \%$ deviation between the experimental and simulated escape times. The results show that the PVP incubated surfaces of our nanofluidic device have slightly lower surface electrostatic potential values in comparison to the previously reported silica-water surface electrostatic potential $\left(2.8 \frac{k_{B} T}{e}\right) .^{5}$

\section{Effect of hydrodynamic coupling between particle and device surfaces}

When a particle moves between parallel surfaces, there is a non-negligible hydrodynamic coupling between the particle and the surfaces that results in an increased friction. Consider a spherical particle of radius $R$ moving at the mid-point position between two parallel surfaces positioned a distance $2 h$ away. Faxén showed that hydrodynamic coupling between the particle and surface leads to a friction factor given by: ${ }^{6}$

$$
\zeta_{\mathrm{eff}}=\frac{\zeta_{0}}{1-1.004(R / h)+0.418(R / h)^{3}+0.21(R / h)^{4}-0.169(R / h)^{5}}
$$

with $\zeta_{0}=6 \pi \eta R$, the friction factor in bulk solution (i.e. far away from the surface). The quantity $\eta$ is the solution viscosity $\left(=8.90 e^{-4}\right.$ Pa.s, i.e. viscosity of water at temperature $298 \mathrm{~K})$. The diffusion coefficient is given by $D=k_{\mathrm{B}} T / \zeta_{\text {eff }}$. 


\section{Relationship Between Zeta Potential and Charge}

Once the size and charge of the spherical particles is known, the particle zeta potential $\zeta$ can be calculated from the below equation: ${ }^{7,8}$

$$
q_{\mathrm{eff}}=-\epsilon_{\mathrm{m}} \epsilon_{0} \kappa 4 \pi R^{2}\left(\frac{k_{B} T}{e}\right)\left[2 \sinh \left(\frac{y}{2}\right)+\left(\frac{4}{\kappa R}\right) \tanh \left(\frac{y}{4}\right)\right]
$$

where $y=\frac{e \zeta}{k_{B} T}$ is the dimensionless zeta potential and $R$ is the particle radius. We numerically invert the equation to access $\zeta$ from knowledge of $R$ and $q_{\text {eff }}$.

\section{Automation}

We automated the measurement approach using a custom Python code to control liddeflection, camera and LED operation (Fig. S7). First, new particles are pumped into the array $(5 \mathrm{~s})$. Then the membrane is deflected by high pressure and the particles are maintained in a state of high confinement. After holding $2 \mathrm{~s}$ to ensure the membrane stabilizes, the applied pressure is decreased to reduce the potential well depth to $D P W_{1}$. After holding another $2 \mathrm{~s}$ to ensure the membrane stabilizes, the microscope camera and LED illumination are turned on and the particle trajectory is recorded for $1 \mathrm{~min}$. Subsequently, the pressure controller reduces the pressure to lower the potential well depth to $D P W_{2}$. Again, after holding $2 \mathrm{~s}$ to ensure the membrane stabilizes, the microscope camera records the particle trajectory for $1 \mathrm{~min}$. Acquisition is then halted and the trajectory saved.

\section{Preparation of Extracellular Vesicles}

EGFRvIII expressing variant of U373 (human astrocytoma) and NHA (normal human astrocytoma) are grown in Dulbecco's modified essential medium (DMEM; Wisent, Canada) supplemented with 10\% heat-inactivated fetal bovine serum (FBS) (Wisent) and 1\% penicillinstreptomycin (Gibco-Life Technologies, Grand Island, NY) at 37 in 5\% CO2. The condi- 


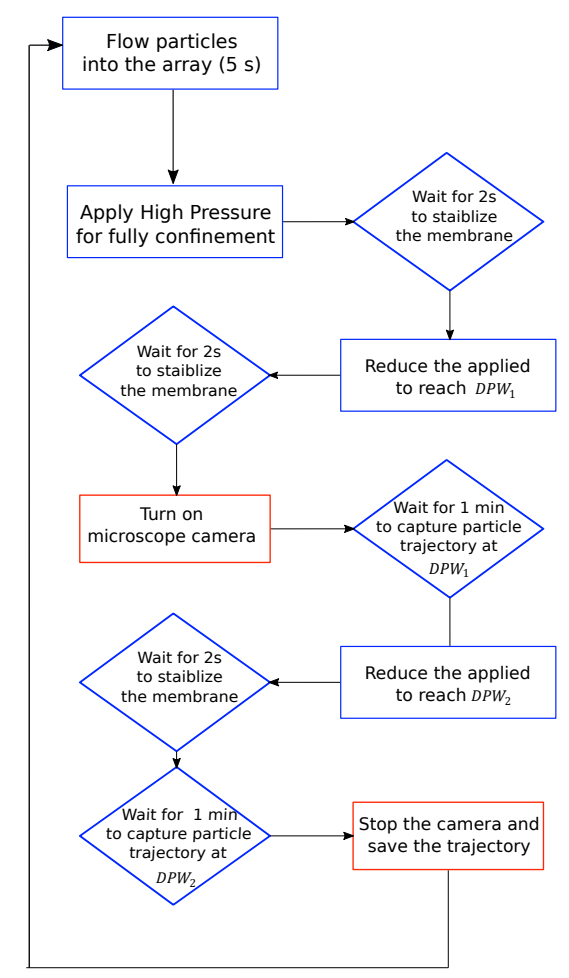

Figure 7: Flow Chart of python code for syncing microscope camera and LED and pressure controller.

tioned medium (CM) was collected from cells grown for 72 hour in culture media containing $10 \%$ of Extracellular Vesicle (EV) depleted FBS (generated by centrifugation at 150,000 $\times g$ for 18 hour at 4 ). The $\mathrm{CM}$ is centrifuged one time at $400 \times 3$ for $15 \mathrm{~min}$ in a filter tube (Merck ${ }^{\top M}$ UFC900324) to concentrate the particles. Finally, the EVs are separated using Size Exclusion Chromatography columns (IZON Science Ltd, United States) in 1mM Tris with $0.4 \mathrm{mM} \mathrm{NaCl}$ solution. For size distribution analysis of EVs, nanoparticle tracking analysis (NTA) was carried out with each collected iodixanol fraction using NanoSight NS500 instrument (NanoSight Ltd., UK). Three recordings of $30 \mathrm{~s}$ at $37^{\circ} \mathrm{C}$ were obtained and processed using NTA software (version 3.0). All experiments were carried out in three biological replicates. 


\section{Data Table for Vesicle Measurements}

Table 1 gives the mean values and accompanying standard deviations for the three vesicle populations measured in the device. Note that the observed difference in values between device class 1 and 2 is not significant.

Table 1: The mean diameter, charge and zeta potential for vesicles measured in the device along with their accompanying standard-deviation $(\sigma)$. In total, 30 liposomes were measured (device class 1) and 100 U373 and NHA EVs each (70\% of EVs in device class 1 for both U373 and NHA, 30\% in device class 2). Each mean value is given an error bar determined from the standard-deviation of the mean.

\begin{tabular}{l|c|c|c|c|c|r} 
Vesicle & $\langle d\rangle(\mathrm{nm})$ & $\sigma_{d}(\mathrm{~nm})$ & $\langle q\rangle(\mathrm{e})$ & $\sigma_{q}(\mathrm{e})$ & $\langle\zeta\rangle(\mathrm{mV})$ & $\sigma_{\zeta}(\mathrm{mV})$ \\
\hline Liposomes & $239 \pm 2$ & 8 & $3010 \pm 40$ & 230 & $37.6 \pm 0.4$ & 2 \\
U373 (all) & $163 \pm 4$ & 35 & $1719 \pm 70$ & 701 & $41.3 \pm 0.6$ & 6.0 \\
NHA (all) & $155 \pm 4$ & 39 & $1883 \pm 84$ & 835 & $48.6 \pm 0.6$ & 6.4 \\
U373 (device class 1) & $164 \pm 4$ & 34 & $1706 \pm 83$ & 691 & $41.5 \pm 0.7$ & 5.5 \\
U373 (device class 2) & $161 \pm 7$ & 38 & $1749 \pm 135$ & 738 & $43.9 \pm 1.4$ & 7.5 \\
NHA (device class 1) & $158 \pm 5$ & 40 & $1960 \pm 103$ & 863 & $48.7 \pm 0.7$ & 5.9 \\
NHA (device class 2) & $148 \pm 6$ & 34 & $1705 \pm 137$ & 748 & $48.2 \pm 1.4$ & 7.5
\end{tabular}

\section{Discussion of Technology Scaling}

The time $t_{N}$ to measure $N$ EVs is given by $t_{N}=t_{o}(N / n)$ where $t_{o}$ is the time required to measure EVs in one field of view and $n$ is the number of EVs measured simultaneously in onefield of view. The time $t_{o}$ is determined by the time to flow particles into the array $(5 \mathrm{~s})$, the time of lid-lowering/raising plus stabilization $(6 \mathrm{~s})$ and the two trajectory integration steps required (each $1 \mathrm{~min}$ long). Thus, $t_{o}=131 \mathrm{~s}$. How many EVs can we measure simultaneously in a field of view? In order to measure the escape time, we need in principle only two adjacent cavities available for a single EV (one well for particle to leave, one well for a particle to enter). Thus, the maximum number of EVs we can fit in principle in array is half the total number of cavities (so the maximum occupancy is 50\%). For cavities with a center-tocenter separation of $3 \mu \mathrm{m}$ in a $100 \times 100 \mu \mathrm{m}^{2}$ field of view, this gives $n=545$, and suggests 
we can measure $1000 \mathrm{EVs}$ in around $4 \mathrm{~min}\left(t_{N=1000}=4 \mathrm{~min}\right)$. Of course, this situation is idealized: we cannot assume the array will be so densely occupied. Yet, note that assuming an occupancy of only $10 \%$ still yields a practical measurement time of around 20 min for 1000 EV's ( $\sim 50 \mathrm{EV} / \mathrm{min})$. Note that multiple occupancy of a well by more than one EV, which might confound the trajectory of different particles, is not an issue: the EVs self-exclude strongly due to their high negative charge. We have never observed multiple occupancy of a well by more than one EV.

In order to increase throughput further we can increase the optical field of view and the corresponding array area, ensuring that a larger number of EVs are measured in parallel for a given acquisition sequence. Single-molecule imaging technology based on back-illuminated CMOS CCDs (such as the Photometrics Prime 95B 25MM and Photometrics Kinetix) routinely provide a $170 \times 170 \mu \mathrm{m}^{2}$ and even $200 \times 200 \mu \mathrm{m}^{2}$ field of view for a $100 \mathrm{x}$ objective. We can further increase the field of view by using a $60 \mathrm{x}$ objective, yielding a $330 \times 330 \mu \mathrm{m}^{2}$ field of view. This increase in the field of view by a factor of 3.3 leads to a 11-fold increase in statistics yielding a $t_{N=1000} \sim 1.8 \mathrm{~min}(\sim 600 \mathrm{EV} / \mathrm{min})$.

Now, of course we cannot exploit the entire array area due to the membrane curvature; we wish to only perform measurements within the "plateau region" where the slit height variation is less than the prescribed value $2 \delta h_{\max }$. Thus, it is important to determine whether it is practical to increase the plateau region to extend across the field of view (i.e. to cover up to a $330 \times 330 \mu \mathrm{m}^{2}$ area). Using a $h_{\max }=0.8 \mathrm{~nm}$, Comsol simulation predicts that a $L_{\text {plateau }}=100 \mu \mathrm{m}$ requires a membrane width of $500 \mu \mathrm{m}$ and a $L_{\text {plateau }}=330 \mu \mathrm{m}$ will require a membrane width of around $800 \mu \mathrm{m}$ (see Fig. S8). While the current class 1 devices with a membrane area of $160 \times 200 \mu \mathrm{m}^{2}$ yield roughly a $L_{\text {plateau }}=15 \mu \mathrm{m}$, we feel that it is quite practical in a next generation of devices to increase the membrane area to $800 \mu \mathrm{m}$, and even higher, as the $100 \mathrm{~nm}$ thick nitride lids are extremely stable. Note that if membrane fragility were a problem, we could simply increase the membrane thickness. If, for some reason we could not make larger stable free standing membranes, than in principle the CLIC approach 
could be used as an alternative, which implements the confinement variation by deforming a coverslip via a piezo-controlled lens-pusher. ${ }^{9}$

Finally, our estimate of only a $10 \%$ array occupancy is quite conservative. We believe it may be feasible to increase array occupancy by increasing sample concentration. Concentration might be performed on-chip, for example, by running EV-containing buffer against a slit barrier with a gap that permits buffer to escape but traps EVs. Note that just a two-fold increase in occupancy (corresponding to only $20 \%$ of all cavities occupied in the array, which is still conservative) will yield a throughput $\sim 1000 \mathrm{EV} / \mathrm{min}$.

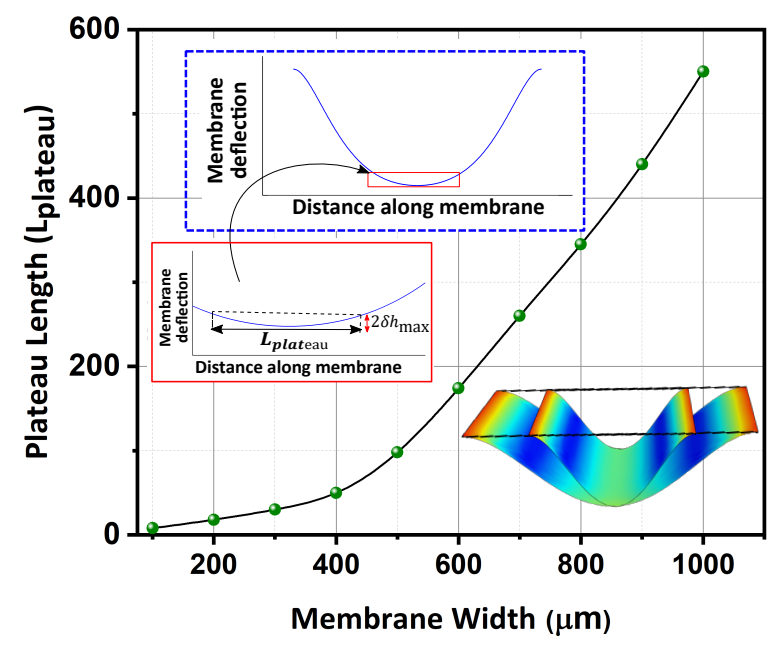

Figure 8: COMSOL simulation results for the plateau length $L_{\text {plateau }}$ versus membrane width. The effective utilizable membrane area is given by $L_{\text {plateau }} \times L_{\text {plateau }}$. The total variation in slit height across the plateau region is given by $2 \delta h_{\max }$. For these simulations $\delta h_{\max }=0.8 \mathrm{~nm}$.

\section{Supporting Movie}

The movie shows the motion of a single YOYO-1 labeled pBR322 vector at different levels of confinement including low confinement $(2 h=188 \mathrm{~nm})$, medium confinement $(2 h=185$ $\mathrm{nm})$ and strong confinement $(2 h=170 \mathrm{~nm})$. In all experiments, the cavity radius $\left(R_{\mathrm{c}}\right)$ is $500 \mathrm{~nm}$ (Figure 2, manuscript). The movement of the plasmid vector is captured using 
fluorescence microscopy and superimposed on graphical images of the nanocavities. The dashed circles depict the escape boundary $\left(r_{\mathrm{esc}}=750 \mathrm{~nm}\right)$. The right panels display the particle status (e.g. whether the particle is inside or outside of the cavities). If the degree of confinement is increased the particle will stay longer in the potential wells. At the highest level of confinement the particle will stay in a single cavity for the entire movie duration. The movie is sped-up by a factor of 2.4 .

\section{References}

(1) Capaldi, X.; Liu, Z.; Zhang, Y.; Zeng, L.; Reyes-Lamothe, R.; Reisner, W. Probing the organization and dynamics of two DNA chains trapped in a nanofluidic cavity. Soft matter 2018, 14, 8455-8465.

(2) Hidrovo, C. H. 2D thickness and temperature mapping of fluids by means of a two-dye laser induced fluorescence ratiometric scheme. Journal of Flow Visualization and Image Processing 2002, 9 .

(3) Krishnan, M. Electrostatic free energy for a confined nanoscale object in a fluid. The Journal of chemical physics 2013, 138, 114906.

(4) Newman, J. Dynamic light scattering as a probe of superhelical DNA-intercalating agent interaction. Biopolymers: Original Research on Biomolecules 1984, 23, 1113-1119.

(5) Ruggeri, F.; Krishnan, M. Lattice diffusion of a single molecule in solution. Physical Review E 2017, 96, 062406.

(6) Happel, J.; Brenner, H. Low Reynolds number hydrodynamics: with special applications to particulate media; Springer Science and Business Media: Berlin, 2012.

(7) Loeb, L., A; Overbeek, J. T. G.; Wiersema, P. H. The electrical double layer around a spherical colloidal particle; MIT Press: Cambridge, 1961. 
(8) Krishnan, M.; Mojarad, N.; Kukura, P.; Sandoghdar, V. Geometry-induced electrostatic trapping of nanometric objects in a fluid. Nature 2010, 467, 692-695.

(9) Berard, D. J.; Miçhaud, F.; Mahshid, S.; Ahamed, M. J.; McFaul, C. M. J.; Leith, J. S.; Bérubé, R., Pierre; Sladek; Reisner, W.; Leslie, S. R. Convex lens-induced nanoscale templating. Proced. Nat. Acad. Sci 2014, 111, 13295-13300. 